\title{
Characterization of StyR-234 non-coding RNA in regulation of Multi-drug resistance in Salmonella enterica serovar typhi
}

\author{
Siventhiran Balakrishnan ${ }^{1}$, Sheryna Kaur ${ }^{1}$, Kishan raj $^{1}$, Sunitha Paripelli ${ }^{2}$, \\ Sathyanarayana Namani ${ }^{2}$, Suresh V. Chinni ${ }^{2}$ \\ ${ }^{1}$ Faculty of Applied Sciences, AIMST University, 08100, Bedong, Kedah. \\ ${ }^{2}$ Faculty of Medicine, AIMST University, 08100, Bedong, Kedah.
}

\begin{abstract}
Non-protein coding RNAs (npcRNA) have been recently recognized in bacterial pathogens as a new group to regulate multi-drug resistance. Among several pathogenic bacterial spcecices, Salmonella enterica serovar Typhi (S. typhi) one of the focusing target in order to prevent severe impact in human health and it pose a problem by causing high fever. we report the characterization of StyR-234, a small antisense $n p c R N A$, discovered from Salmonella typhi. StyR-234 is located on positive strand and antisense to, bacA gene. Since bacA is known to participate in MDR, we hypothesis StyR-234 is involve in bacA regulation thus could have role in drug resistance in $S$. typhi. Overexperssion analysis show that $S$. typhi became more susceptible to six antibiotics (Amikacin, Cefepime, Trimethoprim Sulphamethoxazole, Imipenem, Meropenem, Piperacillin Tazobactam) while more resistance to two antibiotics (Ampicillin and Cefotaxime). And no changes in sensitivity for three antibiotics (Ciprofloxacin, Clindamycin and Ceftazidime). The results indicated that StyR234 increasing susceptibility to many antibiotics could be by down regulating antibiotic resistant gene bacA.
\end{abstract}

KEYWORDS: StyR-234; overexpression; drug resistance; S. Typhi

\section{INTRODUCTION}

Salmonella is categorised under the genus of Enterobacteriaceae that fall in the group of enteric bacteria. They have been very well-studied with respect to virulence mechanisms, microbial pathogenesis, evolution of the genome and many fundamental pathways of gene expression and metabolism. Based on DNA sequences, the Salmonella genus could be categorized into two species, S. enterica and S. bongori, which are further subdivided into subspecies and serovars (>2500 serovars). Salmonella enterica serovar Typhi (from here we will refer to as $S$. typhi), is a Gram negative human specific pathogen causing enteric typhoid fever, an acute life-threatening febrile illness that affects the reticuloendothelial system. According to World Health Organisation (WHO), 16-33 million cases and 500,000-600,000 deaths are reported around the world annually ${ }^{1-}$ ${ }^{3}$. S. typhi is characterized by its flagellar antigen H, its lipopolysaccharidic O antigen and, in addition, it's PS capsular Vi (for virulence) antigen, found at the surface of freshly isolated strains. One of the key features of $S$. typhi is its invasive nature and restriction to human host only. The human host restriction has inhibited direct studies of pathogenicity of $S$. typhi. Thereby most of the understanding of S. typhi has been achieved from comparative research with its closer serovar, S. typhimurium, the mouse model. One of the foremost obstacles to administering effective treatment against Salmonella infection is antibiotic resistance among disease causing strains ${ }^{4}$. The emergence of multidrug resistance (MDR) isolates to all first line drugs such as chloromphenicol, trimethoprim, sulphamethoxazole and ampicillin simultaneously has led to large epidemics and complicated the management of this serious disease ${ }^{5}$.

The emergence of ESBL producers along with multidrug-resistant (MDR) isolates poses a serious problem in the hospital settings ${ }^{6}$. Although there are new agents within existing classes of antimicrobials, currently there are no new classes of antimicrobials in the later phases of development with activity against MDR bacteria ${ }^{7}$. A major challenge in achieving their eradication is a better understanding of bacterial pathogenesis processes ${ }^{8}$. RNAs are also emerging as regulators, enabling the pathogen to adapt its metabolic needs during infection and to express its virulence genes when required.

There are two types of RNA molecules which are expressed in a cell: messenger RNAs (mRNAs) and non-protein-coding RNAs (npcRNAs). The first type provides templates for protein synthesis and the second does not encode proteins but rather exerts various functions at the level of RNA itself. In bacteria, npcRNAs mostly function as coordinators of adaptation processes in response to environmental changes, integrating 
environmental signals and controlling target gene expression ${ }^{9-11}$. During recent years, experimental RNomics revealed the involvement of a number of npcRNAs in the pathogenesis of different virulence bacteria including Staphylococcus aureus, Streptococcus pyogenes, Clostridium perfringens, Salmonella typhimurium, Vibrio cholerae, Pseudomonas aeruginosa ${ }^{12-28}$.

Multidrug resistance in Salmonella enterica serovar Typhi ( $S$. Typhi), the causative agent of typhoid fever remains a global health concern especially in developing countries where inadequate water supply and improper sanitation is prevalent. The emergence of Multidrug Resistant (MDR) strain [defined as strains that confers resistance to all three first line antimicrobials] in late 1980's caused the switching from this first line antimicrobials to fluoroquinolones (FQs) as its primary therapy ${ }^{29}$. However, extensive usages of FQs have led to treatment failure which in turn, brought expanded- spectrum cephalosporins and macrolides as the alternative drugs for the treatment of typhoid fever ${ }^{30}$. Unfortunately, there are also reports of few $S$. Typhi strains, with extended-spectrum $\beta$-lactamases exhibiting resistance to cephalosporin ${ }^{31,32}$, which makes the situation even worst and therefore creates the need to reveal the molecular basis of this phenomenon.

Hitherto, many studies have been conducted to understand the molecular mechanisms underlying bacterial intrinsic resistance but they were largely focussed on regulation at the gene and protein level which is mediated by gene operons or other transcription factors ${ }^{33,34}$. Findings from these studies show that increased efflux pump expression ${ }^{35,36}$, target gene mutation ${ }^{37-40}$ and plasmid-mediated resistance ${ }^{41-43}$ are the major contributors of drug resistance in Salmonella. As a consequence, very few studies were devoted to understand the roles of newly discovered class of gene expression regulators such as non-protein coding RNAs (npcRNAs) in mediating drug resistance ${ }^{44}$. The npcRNAs, which are also widely known as small RNAs (sRNAs), are short RNA molecules that ranges between $50-300$ nucleotides in length ${ }^{45}$ and do not get translated into proteins. There are increasing evidences that show potential role of this group of RNAs in mediating bacterial drug resistance. For an example, the overexpression of a sRNA called DsrA caused multidrug resistance in Escherichia coli by regulating the expression of MdtEF drug efflux pump ${ }^{46}$. Besides that, MicF and MicC sRNAs are shown to regulate drug resistance by controlling the expression of bacterial outer membrane proteins $\mathrm{OmpF}$ and OmpC respectively ${ }^{47-49}$.

Interestingly, in a recent work by our group which identified 97 novel npcRNA candidates from the transcriptome of $S$. Typhi ${ }^{50}$, we postulated that one particular candidate called StyR-234 could be involved in the regulation of the pathogens' drug resistance mechanism. This postulation was made based on the genomic localization of this candidate antisense to the gene which encodes undecaprenyl pyrophosphate phosphatase; a recognized antibiotic resistant protein. Previous studies show that Simultaneous inactivation of $b a c A, y b j G$, and $p g p B$ is lethal in E. coli. Therefore, this prompted us to study the effect of StyR-234 overexpression on the development of drug resistance phenotype of $S$. Typhi.

\section{MATERIALS AND METHODS:}

Conservation analysis and multiple alignments: The sequence conservation of StyR-254 sequence was analyzed using NCBI-BLAST and aligned using CLUSTALW software.

Bacterial strains, plasmids and growth conditions - Bacterial strains and plasmids used in this study are

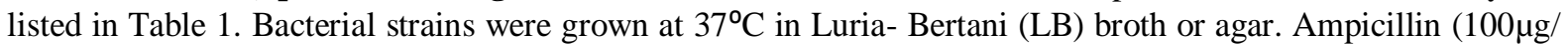
$\mathrm{ml}$ ) were added when required.

\section{Expression analysis of StyR-234}

StyR-234 coding sequence was PCR amplified from the genomic DNA of $S$. Typhi using the primers listed in Table 2, which introduced XbaI and HindIII sites at the ends of the amplified fragment. This fragment was cloned between XbaI and HindIII sites of PCR 2.1 TOPO Cloning vector (Invitrogen) to produce plasmid pStyR234. To over-express StyR-234, pStyR254 was transformed into $S$. Typhi via heat-shock.

Transformation of bacteria: Competent cells of $S$. Typhi were prepared using $1 \mathrm{M}$ calcium chloride. The plasmids PCR 2.1 TOPO vector and pStyR234 were separately transformed in to $S$. Typhi competent cells by giving heat-shock at $42^{\circ} \mathrm{C}$ for 45 seconds. The resulted bacteria were screened by growing on antibiotic plates and PCR. The resulted strains named as ST001+pEmp and ST001+pStyR234.

Induction of gene and harvestion the cells: Bacteria strains ( $S$. Typhi ST001, $S$. Typhi ST001 + pStyR234) were grown in LB broth at $37^{\circ} \mathrm{C}$, shaking at $200 \mathrm{rpm}$. Ampicillin $(100 \mu \mathrm{g} / \mathrm{ml})$ and $1 \mathrm{mM}$ IPTG (Isopropyl- $\beta$-Dthiogalactopyranoside) were added to induce the gene. Cells ( $S$. Typhi + pStyR234) were induced at different phases and harvested at different phases by centrifugation as follows. Cells induced at lag phase and harvested 
at two growth stages, exponential and stationery; cells were induced at exponential phase and harvested at stationery; cells were induced immediate after inoculation and harvested at stationery;

RNA extraction and Northern blot analysis: Total RNA was extracted using Trizol reagent (Invitrogen), according to the manufacturer's instructions. The extracted total RNA was used for Northern blot analysis for StyR-234 npcRNA expression study. Total RNAs $(10 \mu \mathrm{g})$ were fractionated electrophoretically in $8 \%$ Polyacrylamide-7M Urea gels and electrotransferred through semi-dry transfer onto positively charged nylon membranes (Ambion). StyR-234 oligonucleotide was labelled at the 5 '-end with ${ }^{32} \mathrm{P}$ using T4- polynucleotide kinase. The membranes were hybridized with the labelled oligonucleotide in Northern hybridization buffer (Sodium dodecyl sulfate, $\mathrm{Na}_{2} \mathrm{HPO}_{4}$ and $\mathrm{NaH}_{2} \mathrm{PO}_{4}$ ) at $58^{\circ} \mathrm{C}$ overnight and washed with northern washing buffer. The membranes were then exposed to Kodak MS-1 film for 1-3 days.

Disk diffusion assay - In order to determine the phenotypic effect of StyR-234 overexpression, antibiotic susceptibilities were determined for $S$. Typhi ST001, $S$. Typhi ST001 + pStyr254 and S. Typhi ST001 + pEmp using Kirby Bauer disk diffusion method. The test was performed according to the CLSI guidelines. Commercially available antibiotic disks that were used in this test include Ampicillin (10 $\mu$ g), PiperacillinTazobactam $(10 \mu \mathrm{g})$, Amikacin $(30 \mu \mathrm{g})$, Ciprofloxacin $(5 \mu \mathrm{g})$, Cefepime $(30 \mu \mathrm{g})$, Cefuroxime $(30 \mu \mathrm{g})$, Ceftazidime $(30 \mu \mathrm{g})$, Trimethoprim Sulphamethoxazole $(25 \mu \mathrm{g})$, Meropenem $(10 \mu \mathrm{g})$, Clindamycin $(2 \mu \mathrm{g})$ and Imipenem $(10 \mu \mathrm{g})$. Escherichia coli ATCC 25922 was utilized as the control for potency of antibiotic disks.

\section{RESULTS AND DISCUSSION}

Conservation analysis of StyR-234:

In our previous study Chinni et al., StyR-234 was reported as a antisense npcRNA (Fig1). In order to understand the conservation, we performed a BLAST analysis, which proved that StyR-234 sequence is conserved in all Salmonella with percentage of identities ranging from $99 \%$ to $100 \%$. A sequence alignment showing the conservation of StyR-234 sequence is presented in figure 2. StyR-234 is conserved in many of Gram negative bacteria with the range of $70 \%$ to $100 \%$. Interestingly it is also conserved in Gram positive bacteria with significant percentage $(\sim 60 \%-70 \%)$ of identities. The fact that this sequence is conserved in Gram positive and Gram negative further suggests that it could exert crucial roles in their metabolism; besides having potential role in regulating multi-drug resistance cascade.
3213563
3213774
3213887
3214384

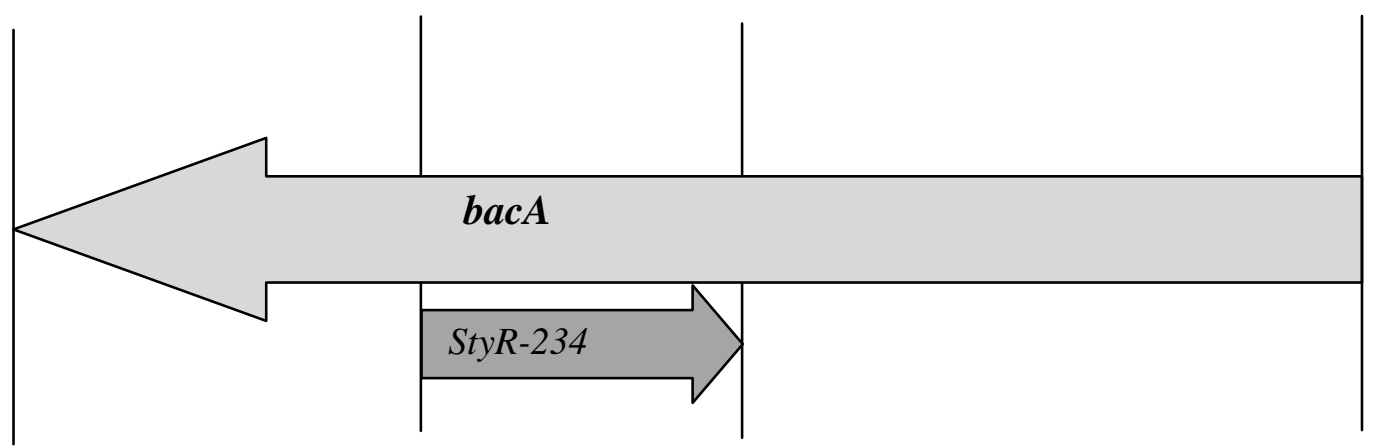

Figure1: Genomic location of StyR-234 in Salmonella Typhi ty2 genome. 


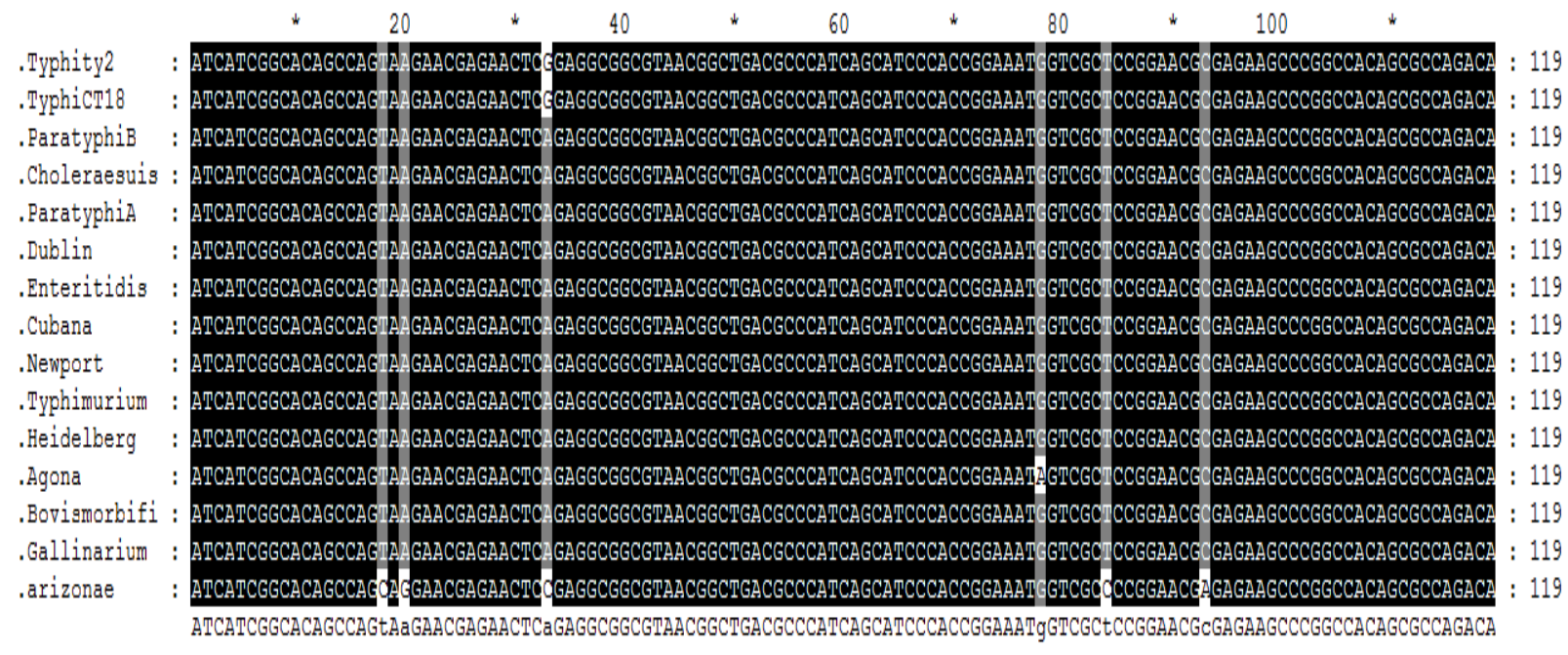

Figure 2: Conservation analysis of StyR-234 sequence in among Salmonella enterica subspecies enterica and arizonae. The species or strain name from the top are Salmonella enterica subspecies enterica serovars Typhi ty2, Typhi Ct18, Paratyphi B, Chleraesius, Paratyphi A, Dublin, Enteritidis, Cubana, Newport, Typhimurium, Heidelberg, Agona, Bovismorbificans, Gallinarium. The last one is the Salmonella enterica subspecies arizonae.

\section{Cloning and over- expression of StyR-234 in S. Typhi ST001}

To evaluate the effect of StyR-234 overexpression in the regulation of $S$. Typhi's MDR cascade, pStyR234 plasmid containing the StyR-234 sequence, was transformed into S. Typhi ST001 cells via heatshock. Total RNA was extracted from 1) $S$. Typhi ST001 2) $S$. Typhi ST001 + pStyR234 (uninduced), 3) $S$. Typhi ST001 + pStyR234 (induced at lag and harvested at stationery) 4) ) S. Typhi ST001 + pStyR234 (induced at exponential and harvested at stationery) 5) S. Typhi ST001 + pStyR234 (induced at lag and harvested at exponential)and 6) ) S. Typhi ST001 + pStyR234 (induced immediate after inoculation and harvested at stationery). Northern analysis was performed to compare the expression level of StyR-234 in the aforementioned samples. As shown in fig 3, StyR-234 is not expressed in exponential phase of S. Typhi ST001, as it expressed only during stationery phase with normal growth conditions. This suggests that StyR-234 could be a growth-phase-dependent npcRNA, which is probably required for the survival of ST001 under nutrient depletion condition.

Interestingly, in the presence of pStyR3, ST001 displayed differential expression of StyR-234; increasing from un-induced to induced,hence, indicated that that the cloning was successful. Apparently, it was evident that the expression of StyR-234 was higher in all growth stages of pStyR234-transformed recombinant ST001 cells upon induction with IPTG compared to the uninduced samples. Interestingly, we noted that the growths of the control cells were also very slow compared to the pStyR3 containing recombinant ST001 cells. This was probably due to the addition of IPTG to the empty vector which could have become toxic to the cell. Overall, the Northern blot result demonstrated that we have successfully cloned and overexpressed the StyR-234 npcRNA

ST001. 


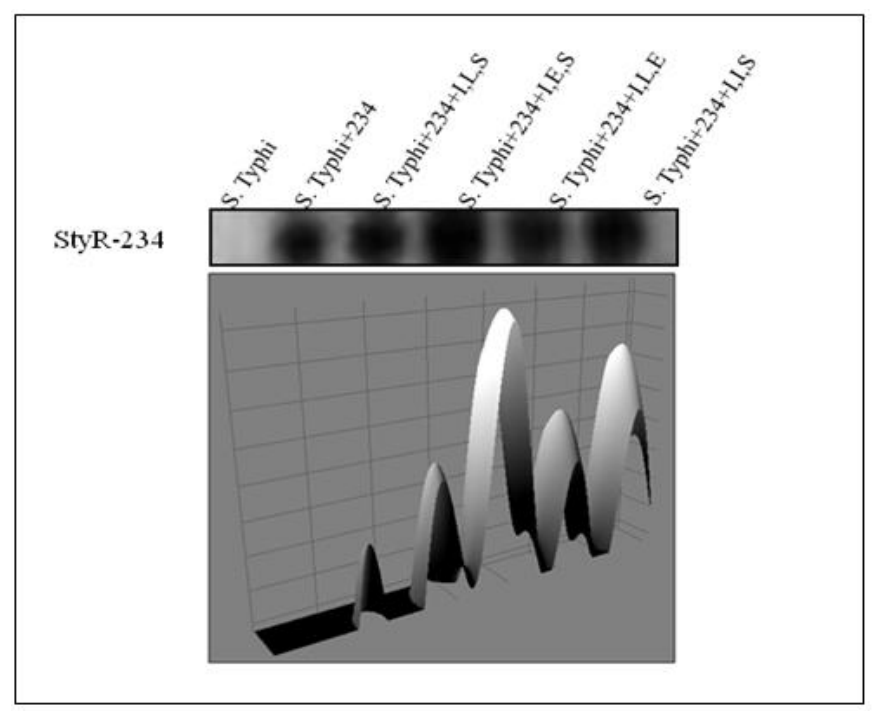

Figure 3 Northern analyses of StyR-234 npcRNA, at different growth stages of S. Typhi ST001 clinical strain and pStyR234-transformed recombinant ST001 strain. Fron left to right, first lane is the S. Typhi ST001 clinical strain exponetial phase, second lane (S. Typhi+234) is the exponential phase of S .Typhi transformed with pSTyR234. Third lane is the S. Typhi transformed with pStyR234 and induced with IPTG at lag phase and harvested at stationery phase. Fourth lane is the S. Typhi transformed with pStyR234 and induced with IPTG at exponetial phase and harvested at stationery phase. Fifth lane (S.Typhi+234+I,L,E) is the S. Typhi transformed with pStyR234 and induced with IPTG at lag phase and harvested at exponential phase. Sixth lane is the S. Typhi transformed with pStyR234 and induced with IPTG immediate after inoculation and harvested at stationery phase.

\section{Overexpression of StyR-233 exerts effect on multi-drug resistance phenotype of $S$. Typhi.}

After successful cloning and over-expression of StyR-234, we performed disk diffusion assay to determine its effect on the drug resistance phenotype of ST001. We found some interesting result when we compared the zone of inhibition of ST001 with the pStyR234-transformed-recombinant ST001. The control strain which possess the empty plasmid was also included in the assay. The assay was performed with 11 types of antibiotic disks, which belongs to 7 different antibiotic groups (Penicillin, Aminoglycosides, Lincosamides, Quinolones, Carbapenems, Cephalosporins 3rd generation, Cephalosporins 4th generation and Sulfonamides).

\begin{tabular}{|l|c|c|c|}
\hline \multicolumn{3}{|c|}{ Zone of inhibition (mm) } \\
\hline \multirow{2}{*}{ Antibiotics } & \multicolumn{3}{|c|}{ Strains } \\
\cline { 2 - 4 } Group A & ST001 & ST001 + pStyR234 & ST001 + pEmp \\
Ampicillin & & & \\
Cefotaxime & 25.33 & 3.50 & 3.00 \\
\hline Group B & 31.00 & 13.67 & 35.67 \\
Amikacin & & & \\
Cefepime & 20.33 & 25.67 & 20.00 \\
Trimethoprim Sulphamethoxazole & 22.67 & 28.67 & 23.30 \\
Imipenem & 31.33 & 33.00 & 30.67 \\
Meropenem & 30.33 & 33.33 & 30.00 \\
Piperacillin Tazobactam & 22.33 & 28.67 & 23.33 \\
\hline Group C & 22.67 & 28.00 & 22.33 \\
Ciprofloxacin & & & 36.00 \\
Clindamycin & 35.33 & 36.00 & 23.00 \\
Ceftazidime & 22.5 & 23.5 & 27.00 \\
\hline
\end{tabular}

Table 1: The zone of inhibition values in $\mathrm{mm}$ with different antibiotics by the recombinant bacteria (ST001+pStyR234) with respect to negative (non-recombinant S. typhi ST001) and positive control (recombinant with empty plasmid: ST001+pEMP) 
According to the result obtained (shown in Table 4 and Figure 4), overexpression of StyR-234 does play role in exerting effects on the bacteria's drug resistance phenotype. This is based on the observation that upon overexpression of StyR-234, there was a decrease in the zone of inhibition of a few antibiotics namely ampicillin, Cefotaxime; suggesting that increased StyR-234 expression leads to reduced susceptibility to these drugs. On the other hand, it was more interesting to discover that there was an increase in the zone of inhibition for majority of the antibiotics tested upon overexpression of StyR-234. These drugs include amikacin, cefepime, imipenem, meropenem, trimethoprim-sulfamethoxazole and piperacillin. This is indeed very interesting as it shows that overexpression of StyR-234 leads to increased susceptibility to all these drugs. On the hand, there were three drugs that did not display much change in zone of inhibition size. These include ciproflaxacin, clindamycin and ceftazidime. The recombinant strain displayed resistance to ampicillin but this should be ignored as it results from the ampicillin resistant gene present in the pStyR234 plasmid.

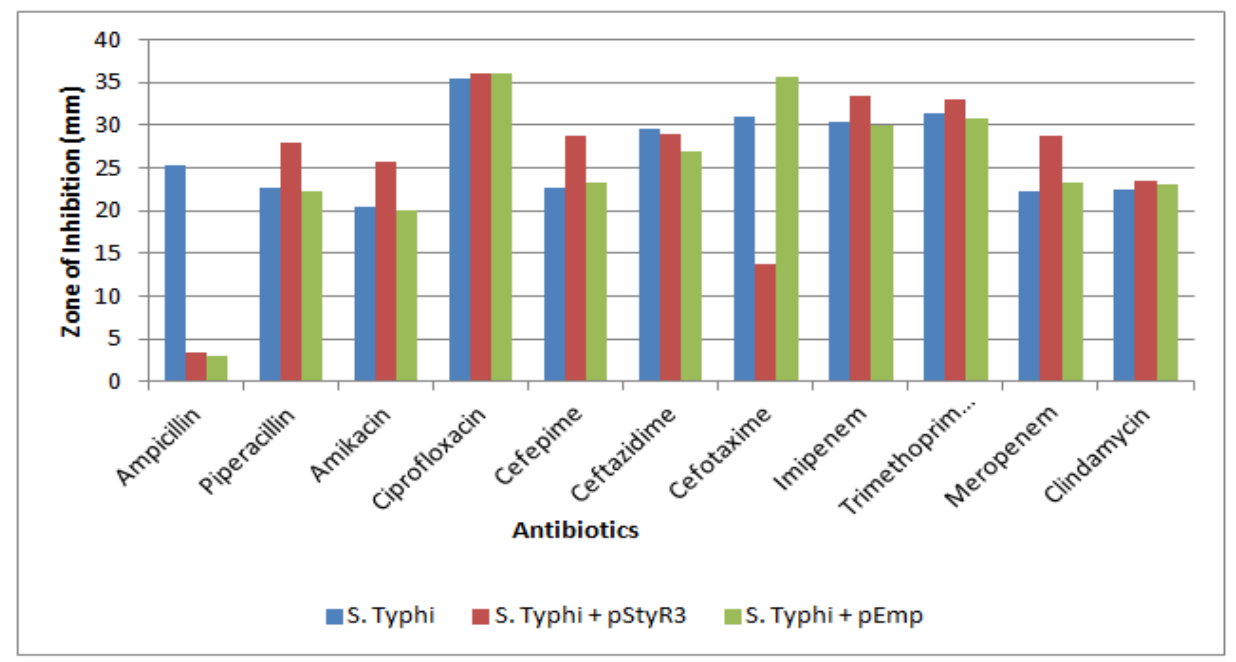

Figure 4: The zone of inhibition values in $\mathrm{mm}$ with different antibiotics by the recombinant bacteria (ST001+pStyR234) with respect to negative (non-recombinant $S$. typhi ST001) and positive control (recombinant with empty plasmid: ST001+pEMP)

Following the identification of 97 novel npcRNA candidates in $S$. Typhi, we are now trying to unveil the function of one particular candidate, called StyR-234. This 119nt-length npcRNA grabbed our attention for further characterization due to its genomic localization; located antisense to a protein-coding gene, bacA which encodes for possible antibiotic resistant protein ${ }^{50,51}$. The bacA gene product is not essential for growth, but the product of the reaction, undecaprenyl phosphate, is essential for the synthesis of peptidoglycan and other cell wall components. At least three additional gene products, YbjG, PgpB, and YeiU, are thought to have undecaprenyl pyrophosphate phosphatase activity in E. coli ${ }^{52}$.

According to the result obtained, StyR-234 was successfully cloned and overexpressed in ST001. Under normal growth condition, StyR-234 was not expressed in all three phases of ST001, but is expressed increasing order from lag to stationery phases in E. coli, hence suggesting that this ncRNA could be important in the survival of the pathogen during nutrient-scarcity ${ }^{50}$.

In contrary, while observing the expression pattern of StyR-234 in the recombinant strain (without induction), it shows that StyR-234 was differentially expressed, increasing from lag to stationery. Meanwhile, in the induced recombinant strain, StyR-234 was expressed in all growth stages and the expression was higher than the un-induced sample, which shows that induction was successful. According to the result observed (Figure 4), StyR-234 over-expression caused reduction in zone size for a few drugs but it displayed increased zone of inhibition in most of the drugs tested. Three of the most notable differences is the difference in the zone size of amikacin, trimethoprim-sulfamethoxazole, cefepime, where the recombinant strain displayed a larger zone compared to ST001 strain. This suggest that overexpression of StyR-234 has the potential to increase the susceptibility of ST001 to these drugs, which previously S. typhi was resistant to. Besides that, overexpression of StyR-234 also caused an increase in the zone size for Imipenem, Meropenem, Piperacillin Tazobactam. This suggests that administration of these drugs can be reconsidered in treatment of typhoid patients. However, the effect of StyR-234 overexpression on the increase of zone size was not very obvious in a few drugs which include ciprofloxacin, clindamycin and ceftazidime. 


\section{CONCLUDING REMARKS}

In summary, in this work, we showed that StyR-234 the over-expression of StyR-234 displayed some changes in the drug susceptibility patterns in ST001. The up-regulation of the StyR-234 leading the bacteria more susceptible to many antibiotics. This susceptibility might be due to the down regulation of the antisense target gene, $b a c A$, which codes for a antibiotic resistance protein.

\section{REFERENCES}

[1]. Zhang XL, Jeza VT and Pan Q. Salmonella typhi: from a human pathogen to a vaccine vector. Cell Mol Immunol, 2008, 5, 91-7.

[2]. Bhan MK, Bahl R and Bhatnagar S. Typhoid and paratyphoid fever. Lancet, 366, 2005, 749-62.

[3]. House D, Bishop A, Parry C, Dougan G. and Wain, J.Typhoid fever: pathogenesis and disease. Curr Opin Infect Dis, 2001, $14,573-8$.

[4]. Cooke FJ andWain J. The emergence of antibiotic resistance in typhoid fever. Travel Med Infect Dis, 2004, 2, 67-74.

[5]. Parry CM. and Threlfall EJ. Antimicrobial resistance in typhoidal and nontyphoidal salmonellae. Curr Opin Infect Dis, 2008, 21, 531-8.

[6]. King Ting Lim, Chew Chieng Yeo, Rohani Md Yasin, Ganeswrie Balan, and Kwai Lin Thong Characterization of multidrug-resistant and extended-spectrum $\beta$-lactamase-producing Klebsiella pneumoniae strains from Malaysian hospitals J. Med. Microbiol. 2009 ,58: 1463-1469

[7]. Arnold RS, Thom KA, Sharma S, Phillips M, Kristie Johnson J, Morgan DJ, Emergence of Klebsiella pneumoniae carbapenemase-producing bacteria. South Med J. 2011 Jan; 104(1):40-5.

[8]. Wassarman KM: Small RNAs in bacteria: diverse regulators of gene expression in response to environmental changes.Cell 2002, 109:141-144.

[9]. Repoila F, Majdalani N, Gottesman S: Small non-coding RNAs,co-ordinators of adaptation processes in Escherichia coli: theRpoS paradigm. Mol Microbiol 2003, 48:855-861.

[10]. Gottesman S: The small RNA regulators of Escherichia coli:roles and mechanisms. Annu Rev Microbiol 2004, 58:303-328.

[11]. Romby P, Vandenesch F, Wagner EG: The role of RNAs in theregulation of virulence-gene expression.Curr Opin Microbiol 2006, 9:229-236.

[12]. Morfeldt E, Taylor D, von Gabain A, Arvidson S: Activation of alpha-toxin translation in Staphylococcus aureus by the transencoded antisense RNA, RNAIII. EMBO J 1995, 14:4569-4577.

[13]. Huntzinger E, Boisset S, Saveanu C, Benito Y, Geissmann T, Namane A, Lina G, Etienne J, Ehresmann B, Ehresmann C et al.:Staphylococcus aureus RNAIII and the endoribonuclease III co-ordinately

[14]. Geisinger E, Adhikari RP, Jin R, Ross HF, Novick RP: Inhibition of rot translation by RNAIII, a key feature of agr function.Mol Microbiol 2006, 61:1038-1048.

[15]. Kreikemeyer B, Boyle MD, Buttaro BA, Heinemann M, Podbielski A: Group A streptococcal growth phase-associated virulence factor regulation by a novel operon (Fas) with homologies to two-component-type regulators requires a small RNA molecule. Mol Microbiol 2001, 39:392-406.

[16]. Li Z, Sledjeski DD, Kreikemeyer B, Podbielski A, Boyle MD: Identification of pel, a Streptococcus pyogenes locus that affects both surface and secreted proteins. J Bacteriol 1999, 181:6019-6027.

[17]. Shimizu T, Yaguchi H, Ohtani K, Banu S, Hayashi H: Clostridial VirR/VirS regulon involves a regulatory RNA molecule for expression of toxins. Mol Microbiol 2002, 43:257-265.

[18]. Ohtani K, Bhowmik SK, Hayashi H, Shimizu T: Identification of a novel locus that regulates expression of toxin genes in Clostridium perfringens. FEMS Microbiol Lett 2002, 209:113-118.

[19]. Heurlier K, Williams F, Heeb S, Dormond C, Pessi G, Singer D, Camara M, Williams P, Haas D: Positive control of swarming, rhamnolipid synthesis, and lipase production by the posttranscriptional RsmA/RsmZ system in Pseudomonas aeruginosa PAO1. J Bacteriol 2004, 186:2936-2945.

[20]. Burrowes E, Abbas A, O’Neill A, Adams C, O’Gara F: Characterisation of the regulatory RNA RsmB from Pseudomonas aeruginosa PAO1. Res Microbiol 2005, 156:7-16.

[21]. Ventre I, Goodman AL, Vallet-Gely I, Vasseur P, Soscia C, Molin S, Bleves S, Lazdunski A, Lory S, Filloux A: Multiple sensors control reciprocal expression of Pseudomonas aeruginosa regulatory RNA and virulence genes. Proc Natl Acad Sci USA 2006, 103:171-176.

[22]. Kay E, Humair B, Denervaud V, Riedel K, Spahr S, Eberl L, Valverde C, Haas D: Two GacA-dependent small RNAs modulate the quorum-sensing response in Pseudomonas aeruginosa.

[23]. J Bacteriol 2006, 188:6026-6033.

[24]. Lenz DH, Miller MB, Zhu J, Kulkarni RV, Bassler BL: CsrA and three redundant small RNAs regulate quorum sensing in Vibrio cholerae. Mol Microbiol 2005, 58:1186-1202.

[25]. Lenz DH, Mok KC, Lilley BN, Kulkarni RV, Wingreen NS, Bassler BL: The small RNA chaperone Hfq and multiple small RNAs control quorum sensing in Vibrio harveyi and Vibrio cholerae. Cell 2004, 118:69-82.

[26]. Altier C, Suyemoto M, Ruiz AI, Burnham KD, Maurer R: Characterization of two novel regulatory genes affecting Salmonella invasion gene expression. Mol Microbiol 2000, 35:635-646.

[27]. Julio SM, Heithoff DM, Mahan MJ: ssrA (tmRNA) plays a role in Salmonella enterica serovar Typhimurium pathogenesis. J Bacteriol 2000, 182:1558-1563.

[28]. Grieshaber NA, Grieshaber SS, Fischer ER, Hackstadt T: A small RNA inhibits translation of the histone-like protein Hc1 in Chlamydia trachomatis. Mol Microbiol 2006, 59:541-550.

[29]. Morita M., Takai N., Terajima J., Watanabe H., Kurokawa M., Sagara H., Ohnishi K., and Izumiya H. 2010. Plasmid mediated resistance to Cephalosporins in Salmonella enterica serovar Typhi. Antimicrob. Agents Chemother. 54(9):3991.

[30]. Threlfall E.J., de Pinna E., Day M., Lawrence J., and Jones J. 2008. Alternatives to ciprofloxacin use for enteric fever, United Kingdom. Emerg Infect Dis. 14(5):860-1.

[31]. Pfeifer Y., Matten J., and Rabsch W. 2009. Salmonella enterica Serovar Typhi with CTX-M $\beta$-Lactamase, Germany. Emerg. Infect Dis. 15:1533-1535.

[32]. Rotimi V.O., Jamal W., Pal T., Sovenned A., and Albert M.J. 2008. Emergence of CTX-M-15 type extended spectrum $\beta$ Lactamase-producing Salmonella spp. In Kuwait and the United Arab Emirates. Journal of Med Microb. 57, 881-886. 
[33]. O’Regan E., Quinn T., Pages J.M., McCusker M., Piddock L., and Fanning S. 2009. Multiple Regulatory Pathways Associated with High-level Ciprofloxacin and Multidrug Resistance in Salmonella enterica Serovar Enteritidis: Involvement of ramA and Other Global Regulators. Antimicrob Agents Chemother. P. 1080-1087.

[34]. Bush K. Alarming $\beta$-Lactamase-mediated resistance in multidrug-resistant Enterobactericeae. 2010. Curr Opin Microbiol. 13(5):558-564.

[35]. Giraud E., Cloeckaert A., Kerboeuf D., Chaslus-Dancla E. 2000. Evidence for Active Efflux as the Primary Mechanism of Resistance to Ciprofloxacin in Salmonella enterica Serovar Typhimurium. Antimicrob Agents Chemother. 44:1223-1228.

[36]. Piddock L.J., White D.G., and Gensberg K. 2000. Evidence for an efflux pump mediating multiple antibiotic resistance in Salmonella enterica serovar Typhimurium. Antimicrob Agents Chemother. 44(11):3118-3121.

[37]. Gaind R., Paglietti B., Murgia M., Dawar M., Uzzau S., Cappuccinelli P., Deb M., Aggarwal P. and Rubino S. 2006. Molecular characterization of ciprofloxacin-resistant Salmonella enterica serovar Typhi and Paratyphi A causing enteric fever in India. J. Antimicrob Chemother. 58(6): 1139-1144.

[38]. Wain J., Hoa N.T., and Chinh N.T., Vinh H., Everett M.J., Diep T.S., Day N.P.J., Solomon T., White N.J., Piddock L.J.V., and Parry C.M. 1997. Quinolone-resistant Salmonella typhi in Vietnam. Molecular basis of resistance and clinical response to treatment. Clin Infect Dis. 25:1404-1410.

[39]. Hirose K., Hashimoto A., Tamura K., Kawamura Y., Ezaki T., Sagara H., and Watanabe H. 2002. DNA sequence analysis of DNA gyrase and DNA topoisomerase IV quinolone resistance-determining regions of Salmonella enterica serovar Typhi and serovar Paratyphi A. Antimicrob Agents Chemother. 46: 3249-3252.

[40]. Piddock L.J., Ricci V., McLaren I. And Griggs D.J. 1998. Role of mutation in gyrA and parC genes in nalidixic-acidresistant salmonella serotypes isolated from animals in the United Kingdom. J. Antimicrob Chemother. 41:635-641.

[41]. Mirza S., Kariuki S., Zamun Z.K., Beeching J. N., and Hart A. C. 2000. Analysis of Plasmid and Chromosomal DNA of Multidrug-Resistant Salmonella enterica serovar Typhi from Asia. J. Clin. Microb. 1449-1452.

[42]. Ling J., and P.Y. Chau. 1984. Plasmid mediating resistance to chloramphenicol, trimethoprim, and ampicillin in Salmonella typhi in the Southeast Asia region. J. Infect. Dis. 149:652.

[43]. Phan M-D., and Wain J. 2008. IncHI plasmids, a dynamic link between resistance and pathogenicity. J. Infect. Dev. Ctries. 2(4): 272-278.

[44]. Yu J., Schneiders T. 2012. Tigecycline challenge triggers sRNA production in Salmonella enterica serovar Typhimurium. BMC Microb. 12:195.

[45]. Storz G., Vogel J., and Wassarman K.M. 2011. Regulation by Small RNAs in Bacteria: Expanding Frontiers. Molecular Cell. 43: 880-891.

[46]. Nishino K., Yamasaki S., Hayashi-Nishino M., Yamaguchi A. 2011. Effect of overexpression of small non-coding DsrA RNA on multidrug efflux in Escherichia coli. J. Antimicrob Chemother. 66(2):291-296.

[47]. Vogel J., Papenfort K. 2006. Small non-coding RNAs and the bacterial outer membrane. Curr Opin Microbiol. 9(6):605-611.

[48]. Delcour A.H. 2009. Outer membrane permeability and antibiotic resistance. Biochim Biophys Acta. 1794(5):808-816.

[49]. Delihas N., Forst S. 2001. MicF: an antisense RNA gene involved in response of Escherichia coli to global stress factors. $J$ Mol Biol. 313(1):1-12.

[50]. Chinni S.V., Raabe C.A., Zakaria R., Randau G., Hoe C.H., Zemann A., Brosius J., Tang T.H., Rozhdestvensky T.S. 2010. Experimental identification and characterization of 97 novel npcRNA candidates in Salmonella enterica serovar Typhi. Nucleic Acid Res. 38(17):5893- 5908.

[51]. El Ghachi M, Bouhss A, Blanot D, Mengin-Lecreulx D (2004). "The bacA gene of Escherichia coli encodes an undecaprenyl pyrophosphate phosphatase activity." J Biol Chem 279(29);30106-13.

[52]. El Ghachi M, Derbise A, Bouhss A, Mengin-Lecreulx D (2005). "Identification of multiple genes encoding membrane proteins with undecaprenyl pyrophosphate phosphatase (UppP) activity in Escherichia coli." J Biol Chem 280(19);18689-95. 